\title{
Colorectal Cancer Surgery during COVID-19 Pandemic in Iran; Most Appropriate Approach
}

\author{
Arash Dooghaie Moghadam, MD'; Pegah Eslami, MD'; Niloofar Razavi-Khorasani, MD'; Bobak Moazzami, MD'; Mostafa Mousavizadeh, \\ $\mathrm{MD}^{2 *}$; Mohammadreza Kalantar Motamedi, $\mathrm{MD}^{3^{*}}$ \\ 'Liver Transplantation Research Center, Tehran University of Medical Sciences, Tehran, Iran \\ ${ }^{2}$ Heart Valve Disease Research Center, Rajaie Cardiovascular Medical and Research Center, Iran University of Medical Sciences, Tehran, Iran \\ ${ }^{3}$ Department of Surgery, Shohada-e-Tajrish Medical Center, Shahid Beheshti University of Medical Sciences, Tehran, Iran
}

T The disease of new coronavirus, known as COVID-19 spread from Wuhan, China, in December 2019. ${ }^{1}$ This severe respiratory disease was recognized as a global pandemic by the WHO on March $11 .^{2}$ In such a crisis, the health status of cancer patients is one of the challenges of the health system.

Today, colorectal cancer (CRC), with $11 \%$ of cancer diagnoses, is the third most common and the second fatal cancer with annually 1.8 million new cases worldwide. In 2018, it caused 881000 deaths, indicating that these patients should not be ignored during the recent pandemic crisis. $^{3}$ In a Chinese study on 1590 COVID-19 patients, 18 patients with a history of cancer had a higher rate of coronavirus infection than normal people. ${ }^{4}$

During this crisis, all international organizations became involved in reducing the burden of disease on active patients and to respond quickly to this epidemic. Making decisions about performing surgery on non-cancerous patients is easy and can be postponed to the post-epidemic conditions. On the other hand, patients requiring emergency surgery such as CRC patients should be treated with the following measures. Numerous guidelines exist for cancer patients undergoing surgery and chemotherapy before the epidemic. Personal care and home quarantine have priority for these patients to reduce the likelihood of infection over the epidemic. Now, what decisions should be made for newly diagnosed CRC patients?

Considering the spreading epidemic and the concern of patients regarding cancer and delay in treatment, postponing surgery to an unknown end of the epidemic seems unreasonable. The long-term outcome of CRC patients is highly dependent on the time of targeted intervention and postponing surgery can cause catastrophic consequences in the patients prognosis.

Here, our concern is for the patients with known cancer who are not affected by coronavirus rather than patients diagnosed with cancer and COVID-19 simultaneously.
The last category can be operated on at coronavirus centers.

Since neoadjuvant treatment is the basis of CRC management, proper and timely decision-making is important. Based on the studies, it was shown that an 8-week postponement after neoadjuvant treatment does not worsen patients' prognosis and decreases the postoperative morbidity in these patients., ${ }^{5,6}$

Those patients who did not undergo any additional neoadjuvant therapy are at increased risk of cancer progression. In our opinion, these patients should be treated immediately after being suspected for COVID-19 infection in order to minimize the morbidity. Overall, we recommend to surgically treat these patients owing to the unknown ending time of the current outbreak. Accordingly, this strategy can ensure rapid control of malignancy and minimize the risk of infections in immunocompromised patients caused by neoadjuvant treatment.

Since a precise treatment guideline for COVID-19 has not been developed yet, it is unknown whether suspected patients who need emergency surgery should also receive COVID-19 treatment immediately after exiting the NPO conditions. Therefore, the decision for performing surgery should be made based on the previous guidelines in order to avoid unnecessary exposure of the surgical team. Since the virus can be found in the blood and stool of COVID-19 patients along with the respiratory system, other modes of disease transmission during surgery should be studied, as well. ${ }^{7}$

In cancers of the perianal region, by forming an abdominal cavity channel, large amounts of aerosol and excretory particles are released in the perineal cavity, increasing the risk of surgeon contamination. Therefore, we recommend to employ a higher standard of protection. Small particles at the site of surgery due to anastomosis, surgical incisions and tissue separation can be absorbed through the surgeon's respiratory system and increase

*Corresponding Authors: Mohammadreza Kalantar Motamedi, MD; Department of Surgery, Shohada-e-Tajrish Medical Center, Shahid Beheshti University of Medical Sciences, Tehran, Iran. Tel: +98-21-88021475; Fax: +98-21-88021475; Email: Itrc.tehran@gmail.com. Mostafa Mousavizadeh, MD; Heart Valve Disease Research Center, Rajaie Cardiovascular Medical and Research Center, Iran University of Medical Sciences, Tehran, Iran. Tel: +98-21-88021475; Fax: +98-2188021475; Email: S.m.mousavizadeh@gmail.com 
the disease risk. ${ }^{8}$ Considering the lack of studies in this regard and the possible existence of blood particles in the resulting smoke in laparoscopic operations, we recommend to perform laparoscopic surgeries by an expert and a negative pressure suction and examine the abdomen for gas leakage and the laparoscopic incision proportional to the operation. ${ }^{9}$ We also recommend to have a gas blowing operation once and not refer the patients to another center for laparoscopic surgery.

The treatment staff's health is the top priority in the whole process of treatment and surgery. The reason is that the tissues sent for pathology and the tissue preserving fluids can be contaminated and thus, the pathology department should be informed of the presence of this specimen. Hence, in our opinion, staff members are recommended to have shift work in the operating room and consider non-invasive and non-surgical procedures for all patients.

In post-operative investigations, considering the prevalence of COVID-19 disease as well as possible abnormal events such as myocarditis in cancer patients, in the case of fever, from our standpoint, the PCR test should also be performed along with common surgical diagnoses including anastomotic leakage.

Another point to consider after surgery is caution with a colostomy, which can be a cause of contamination due to stool removal. So, the patient and the caregiver along with the treatment staff should be cautious and trained.

During the COVID-19 pandemic, the medical staff and especially the operating room staff should receive training in protocols and personal protective equipment (PPE). Operating room personnel and current protocols for cleaning equipment and operating rooms seem to be sufficient given the sensitivity of the virus to solutions such as hypochlorite.

The increasing need for respiratory and ICU equipment during the pandemic is one of the biggest health concerns. In a study in Italy, 20\% of the staff members were infected. ${ }^{10}$ Thus, the hospitalization of these patients and the connection of the physician over follow-up should be performed online, because the hospital itself is one of the places at high risk of transmission. From our point of view, patients admitted to the surgical ward of the hospital must be isolated and frequently visited after surgery as early as possible. Moreover, patients who are susceptible to or contaminated with the COVID-19 need to be separated from other patients. The patients definitely diagnosed with COVID-19 should undergo surgery only in the case of an emergency. Ultimately, it is noteworthy that the health of the treatment staff is the top priority in all stages.

\section{Authors' Contribution}

ADM and PE: designing of the research. BM and NRK: collecting of data. MM and MKM: drafting and finalizing the manuscript.

\section{Conflict of Interest Disclosures}

None.

\section{Ethical Statement}

Not applicable.

\section{References}

1. Zhu N, Zhang D, Wang W, Li X, Yang B, Song J, et al. A Novel Coronavirus from Patients with Pneumonia in China, 2019. N Engl J Med. 2020;382(8):727-33. doi: 10.1056/ NEJMoa2001017.

2. Cucinotta D, Vanelli M. WHO Declares COVID-19 a Pandemic. Acta Biomed. 2020;91(1):157-60. doi: 10.23750/ abm.v91i1.9397.

3. Bray F, Ferlay J, Soerjomataram I, Siegel RL, Torre LA, Jemal A. Global cancer statistics 2018: GLOBOCAN estimates of incidence and mortality worldwide for 36 cancers in 185 countries. CA Cancer J Clin. 2018;68(6):394-424. doi: $10.3322 /$ caac. 21492 .

4. Wang H, Zhang L. Risk of COVID-19 for patients with cancer. Lancet Oncol. 2020;21(4):e181. doi: 10.1016/S14702045(20)30149-2.

5. Des Guetz G, Nicolas P, Perret GY, Morere JF, Uzzan B. Does delaying adjuvant chemotherapy after curative surgery for colorectal cancer impair survival? A meta-analysis. Eur J Cancer. 2010;46(6):1049-55. doi: 10.1016/j.ejca.2010.01.020.

6. Plastiras A, Sideris M, Gaya A, Haji A, Nunoo-Mensah J, Haq A, et al. Waiting Time following Neoadjuvant Chemoradiotherapy for Rectal Cancer: Does It Really Matter. Gastrointest Tumors. 2017;4(3-4):96-103. doi: 10.1159/000484982.

7. Zhang J, Wang S, Xue Y. Fecal specimen diagnosis 2019 novel coronavirus-infected pneumonia. J Med Virol. 2020;92(6):6802. doi: 10.1002/jmv.25742.

8. Liu Y, Song Y, Hu X, Yan L, Zhu X. Awareness of surgical smoke hazards and enhancement of surgical smoke prevention among the gynecologists. J Cancer. 2019;10(12):2788-99. doi: 10.7150/jca.31464.

9. Bree K, Barnhill S, Rundell W. The dangers of electrosurgical smoke to operating room personnel: a review. Workplace Health Saf. 2017;65(11):517-26. doi: 10.1177/2165079917691063.

10. The Lancet. COVID-19: protecting health-care workers. Lancet. 2020;395(10228):922. doi: 10.1016/S01406736(20)30644-9. 